PROCEEDINGS OF THE

AMERICAN MATHEMATICAL SOCIETY

Volume 130, Number 1, Pages 23-32

S 0002-9939(01)06052-X

Article electronically published on May 3, 2001

\title{
NUMERICAL INVARIANTS FOR BUNDLES ON BLOW-UPS
}

\author{
E. BALLICO AND E. GASPARIM \\ (Communicated by Michael Stillman)
}

\begin{abstract}
We suggest an effective procedure to calculate numerical invariants for rank two bundles over blown-up surfaces. We study the moduli spaces $\mathcal{M}_{j}$ of rank two bundles on the blown-up plane splitting over the exceptional divisor as $\mathcal{O}(j) \oplus \mathcal{O}(-j)$. We use the numerical invariants to give a topological decomposition of $\mathcal{M}_{j}$.
\end{abstract}

\section{INTRODUCTION}

Let $X$ be a compact complex surface and let $\pi: \widetilde{X} \rightarrow X$ be the blow-up of $X$ at point $p \in X$. We denote by $\ell$ the exceptional divisor. For simplicity we restrict ourselves to bundles with vanishing first Chern class (although our methods apply to more general situations).

If $E$ is a rank two bundle over a surface $X$ and $j$ is an integer, then a polynomial $p$ determines a bundle $\widetilde{E}$ over the blown-up surface $\widetilde{X}$ such that $\pi_{*} \widetilde{E}^{\vee \vee}=$ $E$ and $\left.\widetilde{E}\right|_{\ell}=\mathcal{O}(j) \oplus \mathcal{O}(-j)$. It follows that we have a correspondence $\{p\} \mapsto$ $\{$ rank two bundle $\widetilde{E} \rightarrow \widetilde{X}\}$ (see [7]). We calculate numerical invariants for $\widetilde{E}$ as a function of the polynomial $p$ and in particular we give an effective procedure to calculate the charge $c_{2}(\widetilde{E})-c_{2}(E)$ which is the difference of second Chern classes. This charge is a sum of two finer invariants $l(Q)$ and $l\left(R^{1} \pi_{*} \widetilde{E}\right)$, where $Q$ is the sheaf defined by the exact sequence $0 \rightarrow \pi_{*} \widetilde{E} \rightarrow\left(\pi_{*} \widetilde{E}\right)^{\vee \vee} \rightarrow Q \rightarrow 0$ (see [2]).

Consider the moduli spaces $\mathcal{M}_{j}$ of bundles on the blown-up plane which split over the exceptional divisor as $\mathcal{O}(j) \oplus \mathcal{O}(-j)$. There is a natural quotient topology on $\mathcal{M}_{j}$ (see [6]) which turns out to be non-Hausdorff. This is to be expected since $\widetilde{\mathbf{C}^{2}}$ is non-compact and there is a priori no notion of stability for bundles over a non-compact space. However, we show that the numerical invariants determine a nice decomposition of $\mathcal{M}_{j}$ into Hausdorff subspaces.

\section{A CORRESPONDENCE BETWEEN POLYNOMIALS AND VECTOR BUNDLES}

Consider a rank two holomorphic bundle $\widetilde{E}$ on $\widetilde{X}$ with vanishing first Chern class. Let $j \geq 0$ be the splitting type of $\widetilde{E}$ over the exceptional divisor, i.e., $\left.\widetilde{E}\right|_{\ell}=\mathcal{O}(j) \oplus \mathcal{O}(-j)$. Then, on a neighborhood $N$ of the exceptional divisor, there is a canonical choice of transition matrix for $\left.\widetilde{E}\right|_{N}$. We may assume $N \simeq \widetilde{\mathbf{C}^{2}}$ (although

Received by the editors September 1, 1999 and, in revised form, May 29, 2000.

2000 Mathematics Subject Classification. Primary 14J60, 14 F05.

The first author was partially supported by MURST (Italy).

The second author was partially supported by CNPQ (Brasil). 
in fact we only need an infinitesimal neighborhood of the exceptional divisor). We fix the coordinates on $N$ putting $N \simeq \widetilde{\mathbf{C}^{2}}=U \cup V$ given by two charts $U=$ $\{(z, u)\} \simeq \mathbf{C}^{2} \simeq V=\{(\xi, v)\}$ with $(\xi, v)=\left(z^{-1}, z u\right)$ on $U \cap V$. In this coordinate system, $\left.\widetilde{E}\right|_{N}$ has a transition matrix of the form

$$
T=\left(\begin{array}{cc}
z^{j} & p \\
0 & z^{-j}
\end{array}\right),
$$

where

$$
p=\sum_{i=1}^{2 j-2} \sum_{l=i-j+1}^{j-1} p_{i l} z^{l} u^{i}
$$

is a polynomial with complex coefficients (see [6]). In other words, $\left.\widetilde{E}\right|_{N}$ is given as an algebraic extension $\left.0 \rightarrow \mathcal{O}(-j) \rightarrow \widetilde{E}\right|_{N} \rightarrow \mathcal{O}(j) \rightarrow 0$ whose extension class is determined by $p$; we will say that $\left.\widetilde{E}\right|_{N}$ is determined by the pair $(j, p)$. It follows that every holomorphic rank two vector bundle over $\widetilde{X}$ with vanishing first Chern class is topologically determined by a triple $(E, j, p)$ (see [7]).

If we fix an integer $j$ and a holomorphic bundle $E$ over $X$, then we get a correspondence between polynomials and bundles on $\widetilde{X}$ :

$$
\begin{aligned}
\{\text { polynomials of the form }(*)\} & \rightarrow\{\text { rank two bundles on } \tilde{X}\} \\
p & \mapsto\left(\begin{array}{cc}
z^{j} & p \\
0 & z^{-j}
\end{array}\right) .
\end{aligned}
$$

Here $i m(\phi)$ consists of all bundles $\widetilde{E}$ over $\widetilde{X}$ satisfying the following two conditions:

८) $\left.\widetilde{E}\right|_{\ell}=\mathcal{O}(j) \oplus \mathcal{O}(-j)$,

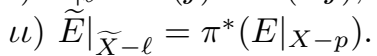

Note that varying $E$ we get all holomorphic bundles over $\widetilde{X}$ which split as $\mathcal{O}(j) \oplus$ $\mathcal{O}(-j)$ over the exceptional divisor and if we also vary $j$ we obtain all rank two holomorphic bundles over $\widetilde{X}$ with vanishing first Chern class (see [7]).

\section{Numerical INVARIANTS}

Let $\widetilde{E}$ be any holomorphic rank two bundle over $\widetilde{X}$ with vanishing first Chern class. In a neighborhood of the exceptional divisor, the bundle $\widetilde{E}$ has a canonical transition matrix $T$, which in turn defines a unique bundle $\widetilde{V}$ over the blown-up plane. On the other hand, if we start with a bundle $\widetilde{V}$ on the blown-up plane and a bundle $E$ over $S$, we can glue them together to form a bundle $\widetilde{E}$ over $\widetilde{S}$ (see [7]). As mentioned in the introduction, the difference of second Chern classes is given by two numerical invariants: $c_{2}(\widetilde{E})-c_{2}(E)=l(Q)+l\left(R^{1} \pi_{*} \widetilde{E}\right)$. But these are local invariants and therefore can be viewed as invariants corresponding to the bundle $\widetilde{V}$ itself.

Recall that we say $\widetilde{E}$ has splitting type $j$ when $\left.\widetilde{E}\right|_{\ell} \simeq \mathcal{O}(j) \oplus \mathcal{O}(-j)$. For splitting types 0 and 1 the moduli spaces $\mathcal{M}_{0}$ and $\mathcal{M}_{1}$ are trivial. In fact, every bundle over $\widetilde{\mathbf{C}^{2}}$ which is trivial over the exceptional divisor is trivial over the entire $\widetilde{\mathbf{C}^{2}}$, hence $\mathcal{M}_{0}$ is just a point; and every bundle over $\widetilde{\mathbf{C}^{2}}$ with splitting type 1 over $\ell$ splits over the entire $\widetilde{\mathbf{C}^{2}}$, therefore $\mathcal{M}_{1}$ is also just a point (see [6]). Therefore the numerical 
invariants are also trivial in these two cases: both invariants vanish for splitting type zero and for splitting type one we have $l\left(R^{1} \pi_{*} \widetilde{E}\right)=0$ and $l(Q)=1$.

Numerical invariants for splitting types 2 and 3 are tabulated below. We leave the calculations for the last section. The notation for the tables is the following. For fixed splitting type, each bundle $\widetilde{V}$ is given as an extension of line bundles with the extension class determined by the polynomial $p$ written in the canonical form $(*)$ as in section 2 . We write the coefficients of $p$ in lexicographical order of the coefficients $p_{i l}$ and denote by $e_{n}$ the monomial corresponding to the $n$-th term. For instance, for splitting type 2 the polynomial written in this order is $p=p_{10} u+p_{11} z u+p_{21} z u^{2}$ and $e_{1}=u, e_{2}=z u, e_{3}=z u^{2}$. We use the term charge for the difference $c_{2}(\widetilde{E})-c_{2}(E)$.

\begin{tabular}{|c|c|c|c|}
\hline TABLE I & \multicolumn{3}{|c|}{ splitting type 2} \\
\hline monomial & $l(Q)$ & $l\left(R^{1} \pi_{*} \widehat{V}\right)$ & charge \\
\hline$e_{1}$ & 1 & 1 & 2 \\
$e_{2}$ & 1 & 1 & 2 \\
\hline$e_{3}$ & 2 & 1 & 3 \\
\hline zero & 3 & 1 & 4 \\
\hline
\end{tabular}

\begin{tabular}{|c|c|c|c|}
\hline TABLE II & \multicolumn{3}{|c|}{ splitting type 3} \\
\hline monomial & $l(Q)$ & $l\left(R^{1} \pi_{*} V\right)$ & charge \\
\hline$e_{1}$ & 3 & 2 & 5 \\
$e_{2}$ & 1 & 2 & 3 \\
$e_{3}$ & 1 & 2 & 3 \\
$e_{4}$ & 3 & 2 & 5 \\
\hline$e_{5}$ & 3 & 3 & 6 \\
$e_{6}$ & 2 & 3 & 5 \\
$e_{7}$ & 3 & 3 & 6 \\
\hline$e_{8}$ & 4 & 3 & 7 \\
$e_{9}$ & 4 & 3 & 7 \\
\hline$e_{10}$ & 5 & 3 & 8 \\
\hline zero & 6 & 3 & 9 \\
\hline
\end{tabular}

\begin{tabular}{|c|c|c|c|}
\hline TABLE III & \multicolumn{3}{|c|}{ splitting type 3} \\
\hline polynomial & $l(Q)$ & $l\left(R^{1} \pi_{*} \widetilde{V}\right)$ & charge \\
\hline$e_{1}+e_{4}$ & 1 & 2 & 3 \\
\hline$e_{4}+e_{5}$ & 2 & 2 & 4 \\
\hline$e_{1}+e_{7}$ & 2 & 2 & 4 \\
\hline
\end{tabular}

\section{Moduli of Bundles on the BlOWn-UP Plane}

Let $\mathcal{M}_{j}$ denote the moduli space of bundles over the blown-up plane $\widetilde{\mathbf{C}^{2}}$ whose restriction to the exceptional divisor equals $\mathcal{O}(j) \oplus \mathcal{O}(-j)$. Our goal here is to show that the two numerical invariants from the previous section provide a decomposition of $\mathcal{M}_{j}$ into Hausdorff subsets.

A remark about the terminology used here is in order. Rigorously we should use the term parameter spaces for the spaces $\mathcal{M}_{j}$, since among other things these spaces are non-Hausdorff and since there is no notion of stable points in this case. 
One possible choice for a notion of stability would be to call a bundle stable when it belongs to the generic subset of $\mathcal{M}_{j}$, which in terms of the numerical invariants corresponds to having the smallest possible invariants. Since this generic part is Hausdorff, these would seem appropriate, but it is not yet clear if this is the best notion of stability to impose.

First we recall the topology of $\mathcal{M}_{j}$. Each bundle $E$ on $\widetilde{\mathbf{C}^{2}}$ with splitting type $j$ is represented by a pair $(j, p)$ where $p$ determines the extension class (see section 2 ). Writing the polynomial $\sum p_{i l} z^{l} u^{i}$ in lexicographical order gives a natural identification of $p$ with the point in $\mathbf{C}^{N}$ whose coordinates are the coefficients of $p$. It is natural to impose the relation $p \sim p^{\prime}$ if $(j, p)$ and $\left(j, p^{\prime}\right)$ represent isomorphic bundles, and take $\mathbf{C}^{N} / \sim$ with the quotient topology. We give $\mathcal{M}_{j}$ the topology induced by the bijection $\mathcal{M}_{j} \rightarrow \mathbf{C}^{N} / \sim$.

It turns out that once this quotient topology is described, a decomposition of the moduli spaces essentially suggests itself, and nicely enough this decomposition is the same as the one we obtain by separating loci of constant pairs of numerical invariants $\left(l(Q), l\left(R^{1} \pi_{*} \widetilde{V}\right)\right)$. In general this decomposition becomes a bit abstract, but it is quite clear in the first examples where we take the splitting type to be a small integer.

4.1. The topological structure of $\mathcal{M}_{2}$. As mentioned in section 3 , the moduli spaces $\mathcal{M}_{0}$ and $\mathcal{M}_{1}$ are trivial; both consist of a single point. The first interesting example happens when the splitting type is 2 , where we have the following structure (see [6]).

The moduli space $\mathcal{M}_{2}$ is isomorphic to $\mathbf{C}^{3} / \sim$ where $\sim$ is the equivalence relation given by $R_{1}$, and $R_{2}$ :

- $R_{1}:\left(p_{10}, p_{11}, p_{21}\right) \sim \lambda\left(p_{10}, p_{11}, *\right)$ if $\left(p_{10}, p_{11}\right) \neq(0,0)$,

- $R_{2}:\left(0,0, p_{12}\right) \sim \lambda\left(0,0, p_{12}\right)$,

where $\lambda \in \mathbf{C}-\{0\}$. This suggests a decomposition $\mathcal{M}_{2}=S_{0} \cup S_{1} \cup S_{2}$ where:

- $S_{0}=\{(0,0,0)\}$,

- $S_{1}=\left\{\left(\lambda p_{10}, \lambda p_{11}, *\right),\left(p_{10}, p_{11}\right) \neq(0,0)\right\} \simeq \mathbf{P}^{1}$,

- $S_{2}=\left\{\lambda\left(0,0, p_{21}\right), p_{21} \neq 0\right\} \simeq\{(0,0,1)\}$.

On the other hand, one could also use the numerical invariants given in Table I to decompose $\mathcal{M}_{2}$. Comparing with Table I we see that the $S_{i} \subset \mathcal{M}_{2}$ with $i=0,1,2$ coincide with the loci of constant $l(Q)$ (or else the loci of constant charge).

4.2. The topological structure of $\mathcal{M}_{3}$. For splitting type 3 the quotient space becomes somewhat more complicated, and both numerical invariants are needed to give a nice decomposition. In the special case of $\mathcal{M}_{2}$ we saw that the decomposition by loci of constant charge already provided us with Hausdorff subspaces $S_{i}$. However, this is in general not the case, as we shall see in the example of $\mathcal{M}_{3}$. In fact, the two finer invariants $l(Q)$ and $l\left(R^{1} \pi_{*} \widetilde{V}\right)$ are needed two give a nice decomposition; or equivalently, one can choose to give the charge and one of the finer invariants.

For splitting type 3 the moduli space is $\mathcal{M}_{3} \simeq \mathbf{C}^{10} / \sim$ with equivalence relation defined by $R_{i}, i=1, \ldots, 6$, below, where $\lambda \in \mathbf{C}-\{0\}$ and $*$ denotes either a complex number or a few complex numbers, on which no restrictions are imposed:

- $R_{1}:\left(a_{1}, \cdots, a_{10}\right) \sim \lambda\left(a_{1}, \cdots, a_{4}, *\right)$ if $\left(a_{2}, a_{3}\right) \neq(0,0)$ or $a_{1}, a_{4} \neq 0$, 
- $R_{2}:\left(a_{1}, 0,0,0, a_{5}, \cdots, a_{10}\right) \sim \lambda\left(a_{1}, 0,0,0, *, a_{7}, *\right)$ if $a_{1} \neq 0$, $\left(0,0,0, a_{4}, \cdots, a_{10}\right) \sim \lambda\left(0,0,0, a_{4}, a_{5}, *\right)$ if $a_{4} \neq 0$,

- $R_{3}:\left(0, \cdots, 0, a_{5}, \cdots, a_{10}\right) \sim \lambda\left(0, \cdots, 0, a_{5}, a_{6}, a_{7}, *\right)$ if $a_{6} \neq 0$ or $a_{5}, a_{7} \neq 0$,

- $R_{4}:\left(0, \cdots, 0, a_{5}, \cdots, a_{10}\right) \sim \lambda\left(0, \cdots, 0, a_{5}, 0,0, *, a_{9}, *\right)$ if $a_{5} \neq 0$, $\left(0, \cdots, 0, a_{7}, \cdots, a_{10}\right) \sim \lambda\left(0, \cdots, 0, a_{7}, a_{8}, *\right)$ if $a_{7} \neq 0$

- $R_{5}:\left(0, \cdots, 0, a_{8}, a_{9}, a_{10}\right) \sim \lambda\left(0, \cdots, 0, a_{8}, a_{9}, *\right)$ if $\left(a_{8}, a_{9}\right) \neq(0,0)$,

- $R_{6}:\left(0, \cdots, 0, a_{10}\right) \sim \lambda\left(0, \cdots, 0, a_{10}\right)$.

This equivalence relation suggests a decomposition of the space $\mathcal{M}_{3}$ into subsets $S_{i}$. Let us look at the most generic part of $\mathcal{M}_{3}$. From the first relation $R_{1}$ it is natural to consider the subset $X_{1}=\mathbf{C}^{10}-V(\mathcal{I})$ where $\mathcal{I}=\left\langle x_{2}, x_{3}, x_{1} x_{4}\right\rangle$. Then the subspace $S_{1}=X_{1} / R_{1}$ is homeomorphic to $\mathbf{C P}^{3}-\{[1,0,0,0],[0,0,0,1]\}$. Comparing with Tables II and III we see that for points in $S_{1}$ the lowest values of the numerical invariants occur. In fact, $S_{1}$ is exactly the locus of points in $\mathcal{M}_{3}$ attaining the lowest invariants.

On the opposite side, relation $R_{6}$ gives us the point $(0, \cdots, 0,1)$ which is the only point of $\mathcal{M}_{3}$ corresponding to charge 8 . There is one point which is more special. The single point $\{0\}$ forms the least generic subset of $\mathcal{M}_{3}$ where the highest values of the numerical invariants are attained.

Among the other relations we notice another interesting fact. Let us look at relations $R_{2}$ and $R_{3}$. From relation $R_{2}$ we obtain a subset $S_{2}$ of $\mathcal{M}_{3}$ homeomorphic to two copies of $\mathbf{C P}^{1}-[0,1]$. On the other hand, from relation $R_{3}$ we obtain a subset $S_{3}$ of $\mathcal{M}_{3}$ homeomorphic to $\mathbf{C P}^{2}-\{[1,0,0],[0,0,1]\}$. Naturally we would like to have $S_{3}$ and $S_{2}$ as separate subsets on a nice decomposition of $\mathcal{M}_{3}$, especially because their union is non-Hausdorff in $\mathcal{M}_{3}$. However, if we were to decompose $\mathcal{M}_{3}$ by loci of constant charge, then $S_{2} \cup S_{3}$ would be contained in the single subset of points corresponding to charge 5 . This makes it evident that one more numerical invariant is necessary to give a nice decomposition of $\mathcal{M}_{3}$, and it is clear that any pair of invariants from Table II distinguishes $S_{2}$ from $S_{3}$.

It comes out nicely that the topological decomposition that is naturally suggested by the description of $\mathcal{M}_{3}$ as a quotient of $\mathbf{C}^{10}$ is exactly the same as the decomposition given by loci of constant pairs of numerical invariants $\left(l(Q), l\left(R^{1} \pi_{*} \widetilde{V}\right)\right)$.

4.3. The topological structure of $\mathcal{M}_{j}$. The facts mentioned about the topology of $\mathcal{M}_{3}$ are readily generalized for higher splitting type. The key fact to have in mind is that to calculate the numerical invariants, one takes into account which are the nonzero coefficients of the polynomial, but not the particular value of each coefficient.

The generic set of $\mathcal{M}_{j}$ is homeomorphic to a complex projective space of dimension $2 j-3$ minus a complex subvariety of codimension at least 2 (see [6]). For points in this generic part, the lower bound of the numerical invariants are attained and these are (see [8]) $l(Q)=1$ and $l\left(R^{1} \pi_{*} \widetilde{V}\right)=j-1$, hence charge $j$. The least generic point in $\mathcal{M}_{j}$ comes from $0 \in \mathbf{C}^{N}$ which by the correspondence from section 2 gets translated into the vanishing of the extension class, that is, to the split bundle $\mathcal{O}(j) \oplus \mathcal{O}(-j)$, for which we have (see [8]) $l\left(R^{1} \pi_{*} \widetilde{V}\right)=j(j+1) / 2$ and $l(Q)=j(j-1) / 2$ and hence charge $j^{2}$. Every intermediate value of the numerical invariants occur for some point in $\mathcal{M}_{j}$ (see [1]). To show that these invariants provide a nice decomposition of $\mathcal{M}_{j}$ into Hausdorff subspaces, we use induction over $j$ together with the fact that there is a topological embedding $\mathcal{M}_{j-1} \hookrightarrow \mathcal{M}_{j}$ (see [9]). 
We then have a topological decomposition of $\mathcal{M}_{j}$ into a union of subspaces homeomorphic to open subsets of complex projective spaces $\mathbf{C P}^{n}$ with $0 \leq n \leq 2 j-3$ and two points. In other words, we have just showed:

Theorem 4.1. The numerical invariants $\left(l(Q), l\left(R^{1} \pi_{*} \widetilde{V}\right)\right)$ provide a decomposition $\mathcal{M}_{j}=\bigcup S_{i}$ where each $S_{i}$ is homeomorphic to an open subset of a complex projective space of dimension at most $2 j-3$. The lower bounds for these invariants are $(1, j-1)$ and this pair of invariants takes place on the generic part of $\mathcal{M}_{j}$ which is homeomorphic to $\mathbf{C} \mathbf{P}^{2 j-3}$ minus a closed subvariety of codimension at least 2 . The upper bounds for these invariants are $(j(j-1) / 2, j(j+1) / 2)$ and this pair occurs at one single point of $\mathcal{M}_{j}$ which represents the split bundle.

\section{Computing the invariants}

The aim of this section is to give a more concrete feeling about the invariants, their geometric meaning and how to calculate them. We remark that the calculations follow an algebraic procedure which has as initial data just the transition matrix $T$ for the bundle $\widetilde{V}$ on a neighborhood of the exceptional divisor. Given this data one calculates the zero-th and first cohomology groups for $\widetilde{V}$ and then it is a matter of simple algebra to calculate $l(Q)$ and $l\left(R^{1} \pi_{*} \widetilde{V}\right)$. Despite the fact that the calculations are long, they are quite simple. In principle one could write a computer program to do them, and it would be interesting to tabulate the invariants for higher values of the splitting type $j$, as it would give a better feeling for how the Hausdorff subsets of $\mathcal{M}_{j}$ are distributed. Since the sum of these numbers gives the charge, in particular this method gives a completely algebraic procedure to calculate the second Chern class of $\widetilde{V}$ directly from its transition matrix. Calculations for the first Chern class of line bundles from the transition matrix are well known, but for rank two bundles the authors do not know of any reference.

5.1. Geometric meaning. Let us make some comments about the geometric interpretation of these numbers. Recall that these are local invariants, so that the geometric meaning corresponds to the behavior of the bundle $\widetilde{E}$ in a neighborhood of the blow-up. Suppose we are given the transition matrix $T=\left(\begin{array}{cc}z^{j} & p \\ 0 & z^{-j}\end{array}\right)$ for $\widetilde{V}$. The data on this matrix means that $\widetilde{V}$ is given as an extension of line bundles $0 \rightarrow \mathcal{O}(-j) \rightarrow \widetilde{V} \rightarrow \mathcal{O}(j) \rightarrow 0$ with extension class determined by the polynomial $p$. When $p=0$ the bundle splits and $l\left(R^{1} \pi_{*} \widetilde{V}\right)=j(j-1) / 2$ assumes its maximal value. For the most general cases $p$ is non-zero on the first formal neighborhood and $\widetilde{V}$ belongs to the generic part of $\mathcal{M}_{j}$ in which case $l\left(R^{1} \pi_{*} \widetilde{V}\right)=j-1$ takes the lowest value. The difference $j(j-1) / 2-l\left(R^{1} \pi_{*} \widetilde{V}\right)$ is a measure of "how far" the bundle is from being split.

The invariant $l(Q)$ is the length of the sheaf $Q$ defined by the exact sequence $0 \rightarrow \pi_{*} \widetilde{E} \rightarrow\left(\pi_{*} \widetilde{E}\right)^{\vee \vee} \rightarrow Q \rightarrow 0$. Note that $Q$ is supported only at a point. If $\pi_{*} \widetilde{E}$ is locally free, then $Q$ is trivial and $l(Q)=0$, but this only happens when $j=0$ in which case $\widetilde{E}$ is a pull back; otherwise, $\pi_{*} \widetilde{E}$ is not locally free. The length $l(Q)$ is the dimension of the stalk at this point and it measures "how far" the sheaf $\pi_{*} \widetilde{E}$ is from being locally free, which can also be seen as a measure of "how far" $\widetilde{E}$ is from being a pull back bundle. 
5.2. How to calculate $l(Q)$. Let $M=\left(\pi_{*} \widetilde{V}\right)_{x}^{\wedge}$ denote the completion of the stalk $\left(\pi_{*} \tilde{V}\right)_{x}$ over the blown-up point $x$. Let $\rho$ denote the natural inclusion of $M$ into its bidual $\rho: M \hookrightarrow M^{\vee \vee}$. We want to compute $l(Q)=\operatorname{dim} \operatorname{coker}(\rho)$. By the theorem on formal functions (see 3 )

$$
M \simeq \lim _{\longleftarrow} H^{0}\left(\ell_{n}, \widetilde{V} \mid \ell_{n}\right) .
$$

There are simplifications that make it easy to calculate $M$. For a fixed splitting type $j$ it is sufficient to calculate $H^{0}\left(\ell_{n}, \widetilde{V} \mid \ell_{n}\right)$ for $n \leq 2 j-2$. This follows from the fact that the polynomial $p$ determining the extension class has non-zero coefficients only up to the $(2 j-2)$-nd formal neighborhood. Moreover, the groups $H^{0}\left(\ell_{n}, \widetilde{V} \mid \ell_{n}\right)$ and $H^{0}\left(\ell_{n+1}, \widetilde{V} \mid \ell_{n+1}\right)$ for $n \geq 2 j-2$ have the same generators as $\mathcal{O}_{x}$-modules. It follows that to determine $M$ it suffices to calculate $H^{0}\left(\ell_{2 j-2}, \widetilde{V} \mid \ell_{2 j-2}\right)$, and the relations among its generators under the action of $\mathcal{O}_{x}^{\wedge}(\simeq \mathbf{C}[[x, y]])$. Recall that for $\widetilde{\mathbf{C}^{2}}$ we have fixed the charts $U=\{(z, u)\} \simeq \mathbf{C}^{2} \simeq V=\{(\xi, v)\}$ with $(\xi, v)=\left(z^{-1}, z u\right)$ on $U \cap V$. Since the blow-up map $\pi: \widetilde{\mathbf{C}^{2}} \rightarrow \mathbf{C}^{2}$ is given by $(x, y)=\pi(z, u)=(u, z u)$ on the $U$ chart the natural action of $x$ and $y$ on this space is that $x$ acts by multiplication by $u$ and $y$ acts by multiplication by $z u$.

Calculations of $l(Q)$ for the split case and also for the generic case (corresponding to $p=u$ ) appeared in [8]. Here we present the calculations for the bundle given by transition matrix

$$
T=\left(\begin{array}{cc}
z^{2} & z u^{2} \\
0 & z^{-2}
\end{array}\right)
$$

which is neither generic nor split and corresponds to the subset $S_{2} \subset \mathcal{M}_{2}$. According to our previous remarks it suffices to calculate the $\mathbf{C}[[x, y]]$-module structure of $H^{0}\left(\ell_{2}, \widetilde{V} \mid \ell_{2}\right)$. We denote by $A\left\langle a_{1}, \cdots, a_{k}\right\rangle$ the $A$-module generated by $a_{1}, \cdots, a_{k}$. We claim that $M=\mathbf{C}[[x, y]]\left\langle\alpha_{0}, \beta_{0}, \beta_{1}, \beta_{2}\right\rangle$ where

$$
\alpha_{0}=\left(\begin{array}{c}
u^{2} \\
0
\end{array}\right), \beta_{0}=\left(\begin{array}{l}
0 \\
1
\end{array}\right), \beta_{1}=\left(\begin{array}{l}
0 \\
z
\end{array}\right), \beta_{2}=\left(\begin{array}{c}
-z u^{2} \\
z^{2}
\end{array}\right)
$$

with relations

$$
\left\{\begin{array}{c}
x \beta_{1}-y \beta_{0} \\
x \beta_{2}-y\left(\alpha_{0}+\beta_{1}\right) .
\end{array}\right.
$$

This is calculated as follows. If $s=\left(\begin{array}{l}a \\ b\end{array}\right)$ is a section of $\widetilde{V} \mid \ell_{2}$, then on the $U$ chart $s$ has a power series expression of the form

$$
s=\sum_{i=0}^{2} \sum_{k=0}^{\infty}\left(\begin{array}{l}
a \\
b
\end{array}\right) z^{k} u^{i} .
$$

Changing coordinates we have that

$$
T s=\sum_{i=0}^{2} \sum_{k=0}^{\infty}\left(\begin{array}{c}
z^{2} a_{i k}+z u^{2} b_{i k} \\
z^{-2} b_{i k}
\end{array}\right) z^{k} u^{i}
$$

must be holomorphic in the $V$ chart, i.e., in the variables $\xi$ and $v$. Since $\xi=z^{-1}$ and $v=z u$, this imposes the condition that all elements of $T s$ having terms $z^{n} u^{i}$ with $n>i$ must vanish. Because the second coordinate in Ts has the terms $z^{-2} b_{i k} z^{k} u^{i}$ 
it follows that

$$
b=\sum_{i=0}^{2} \sum_{k=0}^{i+2} b_{i k} z^{k} u^{i} .
$$

Plugging this expression into the first coordinate of $T s$ we have that

$$
\sum_{i=0}^{2} \sum_{k=0}^{2} z^{2} a_{i k}+z u^{2} \sum_{i=0}^{2} \sum_{k=0}^{i+2} b_{i k} z^{k} u^{i}
$$

must be holomorphic on $z^{-1}$ and $z u$. Imposing the vanishing of the terms on $z^{n} u^{i}$ with $n>i$ we get that the expression for $a$ must be

$$
a=a_{20} u^{2}-b_{02} z u^{2} .
$$

Writing out the expression for the section $s$, we have thus concluded that

$$
\begin{aligned}
s & =\left(\begin{array}{l}
a \\
b
\end{array}\right)=b_{00}\left(\begin{array}{l}
0 \\
1
\end{array}\right)+b_{01}\left(\begin{array}{l}
0 \\
z
\end{array}\right)+b_{02}\left(\begin{array}{c}
-z u^{2} \\
z^{2}
\end{array}\right) \\
& +b_{10}\left(\begin{array}{l}
0 \\
u
\end{array}\right)+b_{11}\left(\begin{array}{c}
0 \\
z u
\end{array}\right)+\cdots+a_{20}\left(\begin{array}{c}
u^{2} \\
0
\end{array}\right) .
\end{aligned}
$$

Notice that $\left(\begin{array}{l}0 \\ u\end{array}\right)=x \beta_{0}$ and $\left(\begin{array}{c}0 \\ z u\end{array}\right)=y \beta_{0}$ and similar expressions hold for the terms we omitted. It follows that $H^{0}\left(\ell_{2}, \widetilde{V} \mid \ell_{2}\right)$ is generated as a $\mathbf{C}[[x, y]]$-module by

$$
\alpha_{0}=\left(\begin{array}{c}
u^{2} \\
0
\end{array}\right), \beta_{0}=\left(\begin{array}{l}
0 \\
1
\end{array}\right), \beta_{1}=\left(\begin{array}{l}
0 \\
z
\end{array}\right), \beta_{2}=\left(\begin{array}{c}
-z u^{2} \\
z^{2}
\end{array}\right),
$$

as claimed. Clearly, the relations given hold, and we know the number of relations must equal the number of generators minus 2 because $\widetilde{V}$ has rank 2 . Once $M$ is found, it is a simple algebraic calculation to find the dual and bidual. We have that $M^{\vee}=\langle A, B, C\rangle$ has the generators

$$
A:\left\{\begin{array}{l}
\alpha_{0} \rightarrow x, \\
\beta_{2} \rightarrow y, \\
\beta_{0} \rightarrow 0, \\
\beta_{1} \rightarrow 0,
\end{array} \quad B:\left\{\begin{array}{c}
\alpha \rightarrow 0, \\
\beta_{2} \rightarrow y^{2}, \\
\beta_{0} \rightarrow x^{2}, \\
\beta_{1} \rightarrow x y,
\end{array} \quad C:\left\{\begin{array}{c}
\alpha \rightarrow-y \\
\beta_{2} \rightarrow 0 \\
\beta_{0} \rightarrow x \\
\beta_{1} \rightarrow y
\end{array}\right.\right.\right.
$$

satisfying the relation

$$
y A-B+x C=0 .
$$

$M^{\vee \vee}$ is free on two generators (this will always be the case since it is the stalk of a rank two locally free sheaf). $M^{\vee \vee}=\langle\mathcal{A}, \mathcal{B}\rangle$ where

$$
\mathcal{A}:\left\{\begin{array}{l}
A \rightarrow 1, \\
B \rightarrow y, \\
C \rightarrow 0,
\end{array} \quad \mathcal{B}:\left\{\begin{array}{l}
A \rightarrow 0, \\
B \rightarrow x, \\
C \rightarrow 1 .
\end{array}\right.\right.
$$

The map $\rho: M \rightarrow M^{\vee \vee}$ is given by evaluation. Explicitly

$$
\rho:\left\{\begin{array}{l}
\alpha_{0} \rightarrow x \mathcal{A}-y \mathcal{B} \\
\beta_{2} \rightarrow y \mathcal{A} \\
\beta_{0} \rightarrow x \mathcal{B} \\
\beta_{1} \rightarrow y \mathcal{B} .
\end{array}\right.
$$

Hence $\operatorname{im}(\rho)=\langle x \mathcal{A}-y \mathcal{B}, y \mathcal{A}, x \mathcal{B}, y \mathcal{B}\rangle$, and $\operatorname{coker}(\rho)=\langle\overline{\mathcal{A}}, \overline{\mathcal{B}}\rangle$. Therefore $l(Q)=$ $\operatorname{dim} \operatorname{coker}(\rho)=2$. 
5.3. How to calculate $l\left(R^{1} \pi_{*} \widetilde{V}\right)$. Using the theorem on formal functions we have

$$
l\left(R^{1} \pi_{*} \widetilde{V}\right)=\operatorname{dim} \lim _{\longleftarrow} H^{1}\left(\ell_{n}, \widetilde{V} \mid \ell_{n}\right) .
$$

But because the extension class for $\widetilde{V}$ is given by the polynomial $p$ which has non-zero coefficients only up to the $(2 j-2)$-nd formal neighborhood it suffices to calculate $H^{1}\left(\ell_{2 j-j}, \widetilde{V} \mid \ell_{2 j-2}\right)$. The numerical invariant $l\left(R^{1} \pi_{*} \widetilde{V}\right)$ counts just the number of generators of $H^{1}\left(\ell_{2 j-2}, \widetilde{V} \mid \ell_{2 j-2}\right)$. The calculations of $l\left(R^{1} \pi_{*} \widetilde{V}\right)$ are even simpler than those for $l(Q)$.

We take the same example as in the previous section. Let $\widetilde{V}$ be given by transition smatrix $T=\left(\begin{array}{cc}z^{2} & z u^{2} \\ 0 & z^{-2}\end{array}\right)$. We need to find the generators for $H^{1}\left(\ell_{2}, \widetilde{V} \mid \ell_{2}\right)$. Let $\sigma \in H^{1}\left(\ell_{2}, \widetilde{V} \mid \ell_{2}\right)$. Then $\sigma=\sum_{i=0}^{2} \sum_{z=-\infty}^{\infty}\left(\begin{array}{c}a_{i k} \\ b_{i k}\end{array}\right) z^{k} u^{i}$. But $\sum_{i=0}^{2} \sum_{z=0}^{\infty}\left(\begin{array}{c}a_{i k} \\ b_{i k}\end{array}\right) z^{k} u^{i}$ gives a holomorphic function in $U$ and therefore represents a coboundary, which may subtract from $\sigma$ without changing its cohomology class. Hence

$$
\sigma \sim \sum_{i=0}^{2} \sum_{z=-\infty}^{-1}\left(\begin{array}{l}
a_{i k} \\
b_{i k}
\end{array}\right) z^{k} u^{i} .
$$

Changing coordinates we have $T \sigma=\sum_{i=0}^{2} \sum_{z=0}^{\infty}\left(\begin{array}{c}a_{i k}+z u^{2} b_{i k} \\ z^{-2} b_{i k}\end{array}\right) z^{k} u^{i}$ in which every term is holomorphic in the $V$ chart except for $a_{1,-1} z$. Subtracting the holomorphic terms we are left with $T \sigma \sim\left(\begin{array}{c}a_{1,-1} z \\ 0\end{array}\right)$ where $a_{1,-1} \in \mathbf{C}$. Therefore $\sigma=$ $T^{-1} T \sigma \sim\left(\begin{array}{c}a_{1,-1} z^{-1} \\ 0\end{array}\right)$ and we conclude that $H^{1}\left(\ell_{2}, \widetilde{V} \mid \ell_{2}\right)$ is generated by $\left(\begin{array}{c}z_{0}^{-1} \\ 0\end{array}\right)$ and $l\left(R^{1} \pi_{*} \widetilde{V}\right)=1$.

5.4. General calculation procedure. Summing up, the numerical invariants $l(Q)$ and $l\left(R^{1} \pi_{*} \widetilde{V}\right)$ are calculated from the zero-th and first cohomologies of $\widetilde{V}$ on formal neighborhoods of the exceptional divisor. Since bundles on $\widetilde{\mathbf{C}^{2}}$ with splitting type $j$ are determined by their restriction to the $(2 j-2)$-nd formal neighborhood, it turns out that the invariants are determined by the cohomology groups $H^{0}\left(\ell_{2 j-2}, \widetilde{V} \mid \ell_{2 j-2}\right)$ and $H^{1}\left(\ell_{2 j-2}, \widetilde{V} \mid \ell_{2 j-2}\right)$ which are in fact quite simple to calculate.

Steps to calculate $l(Q)$ : find the generators $\left\{\alpha_{i}\right\}$ of $H^{0}\left(\ell_{2 j-2}, \widetilde{V} \mid \ell_{2 j-2}\right)$, determine the $\mathcal{O}_{x}$-module $M$ generated by the $\left\{\alpha_{i}\right\}$, write the natural inclusion $\rho: M \hookrightarrow M^{\vee \vee}$, then $l(Q)=\operatorname{dim}$ coker $\rho$.

Steps to calculate $l\left(R^{1} \pi_{*} \widetilde{V}\right)$ : find the dimension of $H^{1}\left(\ell_{2 j-2}, \widetilde{V} \mid \ell_{2 j-2}\right)$ as a $k(x)$ vector space, this dimension is $l\left(R^{1} \pi_{*} \widetilde{V}\right)$.

\section{REFERENCES}

[1] Ballico, E. and Gasparim, E. Vector Bundles on a Neighborhood of an exceptional curve and Elementary Transformations, to appear

[2] Friedman R. and Morgan, J. On the Diffeomorphism Types or Certain Algebraic Surfaces II, Journal of Diff. Geometry 27, 371-398 (1988) MR 89d:57047

[3] Hartshorne, R. Algebraic Geometry, Graduate Texts in Mathematics, Springer Verlag (1977) MR 57:3116

[4] Lang, S. Algebra, Addison-Wesley Pub. Co. 3rd. Ed. (1993)

[5] Gasparim, E. Holomorphic bundles on $\mathcal{O}(-k)$ are algebraic, Comm. Algebra, 25(9), 30013009 (1997) MR 98f:14037

[6] Gasparim, E. Rank Two Bundles on the Blow-up of $\mathbf{C}^{2}$, Journal of Algebra, Vol. 199, 581-590 (1998) MR 99a:14063 
[7] Gasparim, E. On the Topology of Holomorphic Bundles, Boletim da Sociedade Paranaense de Matemática, 18, 1-7 (1998) CMP 2000:15

[8] Gasparim, E. Chern Classes of Bundles on Rational Surfaces, Politécnico di Torino, Rapporto Interno N.30 (1998)

[9] Gasparim, E. Moduli of Bundles on the Blown-up Plane, math.AG/9810106

Department of Mathematics, University of Trento, 38050 Povo (TN), Italy

E-mail address: ballico@science.unitn.it

Departamento de Matematica, Universidade Federal de Pernambuco, 50670/901 ReCIFE (PE), BRASIL

E-mail address: gasparim@dmat.ufpe.br 